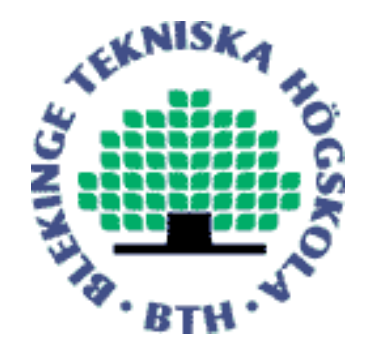

Copyright @ 2011 IEEE.

Citation for the published paper:

Title:

Experimental results of passive imaging of moving continuous broadband sound sources within a sensor field

Author:

Viktoria Tegborg, Mats Pettersson, Ingvar Claesson

Journal:

IEEE journal of oceanic engineering : a journal devoted to the application of electrical and electronics engineering to the oceanic environment

Year:

2011

Vol:

36

Issue:

1

Pagination:

26-37

URL/DOI to the paper:

10.1109/JOE.2010.2066790

This material is posted here with permission of the IEEE. Such permission of the IEEE does not in any way imply IEEE endorsement of any of BTH's products or services Internal or personal use of this material is permitted. However, permission to reprint/republish this material for advertising or promotional purposes or for creating new collective works for resale or redistribution must be obtained from the IEEE by sending a blank email message to pubs-permissions@ieee.org.

By choosing to view this document, you agree to all provisions of the copyright laws protecting it. 


\title{
Experimental Results of Passive Imaging of Moving Continuous Broadband Sound Sources Within a Sensor Field
}

\author{
Viktoria Tegborg, Mats I. Pettersson, and Ingvar Claesson
}

\begin{abstract}
Sparsely distributed sensors or sparse arrays can be associated with high positional accuracy and capability for large area surveillance. This article shows, both through simulations and with real measurement data, that sparse sensor systems can be used to accomplish high-performance underwater surveillance. The article focuses on measurement setups with several passive single hydrophones placed hundreds of meters apart in a water depth of dozen of meters. By such a setup a sound source is then more likely to move inside the array, and thus near-field processing can be considered. The sensor pairs will also be located in different directions in relation to the sound source. These two prerequisites give the possibilities to high spatial resolution. Images of the coherent sound activity for different sensor pairs are formed, and every sensor pair map is summarized into a resulting map. In this way, an arbitrary number of sound sources of a target can be resolved. Good correlation results are shown despite the fact that the sound source is at the same distance as the baseline of the sensor pairs. This is achieved by Doppler compensating for target movement and also by whitening of the cross-spectra. The analyses of the measurements also show that baseline-dependent bandwidth can improve the results. In the paper, it is indicated from experimental data that two propeller sound sources could be resolved, and in another measurement setup the engine could be separated from the propellers.
\end{abstract}

Index Terms-Acoustic imaging, Sonar signal processing, Underwater acoustical measurements

\section{INTRODUCTION}

An increased interest in systems of sparsely distributed sensors or sparse arrays for surveillance has been seen in recent years. This is due to better computational capabilities and also the use of the Global Positioning System (GPS) that has given new opportunities to synchronize beetwen different groups of sensors.

With sensor pairs in different directions and at different ranges, passive systems provide an opportunity to detect, classify and even identify a target's speed and direction without being revealed to the target.

There have been many publications in the field of passive multistatic radar in recent years [1]. In radar applications, the large sparse array will add the capability to detect and measure

The authors are with the Department of Applied Signal Processing, Blekinge Institute of Technology, Ronneby, Sweden, e-mail: viktoria.zetterberg@bth.se.

The authors would like to thank The Knowledge Foundation in Sweden for their sponsorship. Viktoria would also like to thank M.Sc. Lars Tegborg, $\mathrm{CEO}$ at EmbeddedArt for his contribution to this work concerning hyperboloid surfaces, doppler and ambiguity function, proof-reading of matematical expressions, and also for last refinement of graphics. She would also like to thank Associate Professor Lennart Tegborg for his advices of the text's outline and English grammar localization with very high accuracy. In many ways, this can lead to a paradigm shift. One example is in the case of tracking and anti-air missile guidance [2].

In underwater acoustics, the increased processing capabilities have lead to large sparse arrays that can observe all targets in the near-field of the array just by backprojecting the measurement data. Backprojection of data is commonly used for far-field applications in matched field processing and time reversal acoustics. Within these techniques models of the environment are used. For near-field applications, as in this article, the advantage is that no models of the environment have to be considered since the travel path is much shorter and thereby less complicated. Backprojection with one assumed speed of sound of the whole volume can be used.

Within a sparse array, the sound source is also registered from different angles. With sensor pairs in different directions and at different ranges, images of several sound sources can be formed. No assumptions about number of sound sources, their location (near- or far-field), the size of the sound source, or complicated environmental models have to be taken into account. The only parameters need to be found are the speed and velocity.

There are, however, very few publications on underwater sparse arrays in the field of passive acoustical applications, and even fewer papers concerning passive imaging of moving continuous sources in the near-field. In the field of passive acoustical applications, sparse arrays have been used for localization of transients in different applications. For seismology applications, a multistatic system localized and imaged transients in [3]. In [4], clicks from sperm whales were localized by 5 arrays consisting of 5 sensors each spread several kilometers apart in the water and situated at a depth of up to one kilometer.

To the best of our knowledge, no article concerning imaging of broadband moving sound sources has been published. In [5], however, imaging of an underground non-moving source with transients in a frequency range of 5 to $50 \mathrm{~Hz}$, using only two line arrays, was discussed. Furthermore, in [6], simulated broadband non-moving continuous sound sources were imaged, and a comparison of results from 4 different methods was given. An image was defined as a comparison between predicted and measured time lags projected into a map consisting of the horizontal plane around the array. Results from the article indicated that the time of arrival difference (TOAD) method, which is similar to the solution presented in this article, gave the best results for high Signal 
to Noise Ratios (SNR).

In applications of sparse arrays with many hydrophones, [7] is the most similar publication in comparison to the work presented in this paper. In [7], moving broadband targets were localized by 16 hydrophones in a sparse array. The article focused mainly on localization and used a time of arrival method for single targets. No image generation was considered. The advantage of imaging instead of time of arrival methods is that no assumptions about number of sound sources or the location have to be considered.

Thus, to our knowledge, no publications concerning imaging of broadband moving sound sources exists.

In this paper, a method for imaging moving continuous broadband sound sources within a scattered sensor array is demonstrated. The image is defined as backprojected pairwise coherence over an area in the water volume, where different sensor pairs record different sides of the sound sources.

By using filtered and weighted correlation results from all sensor pairs, the image quality of the sound source can be improved significantly. In this paper, we use an integration time such that the synthetic aperture formed by the target's motion is small. However, thanks to the large amount of sensors and the measuring speed and direction, the three-dimensional (3D) speed and velocity can be found with the help of the overdetermined equation system. In this way, it could be possible to increase the integration time by increasing the compensation for the range walk or range bending (Doppler changes) in the signals. This is associated with passive synthetic aperture sonar, and a larger resolution in all dimensions could be found. The higher resolution for each sensor leads to higher resolution and better quality in the final image. This image can be used as an instrument for detection, localization, classification, and identification.

By placing sensors over the entire surveillance area, the system gets closer to the target under observation, and complicated propagation condition effects, typical for those in shallow waters, lessen. The disadvantage of this is that the received signals then become more dissimilar to each other and thereby the coherence is decreased.

In a previous work [8], real measurements from three different baselines were used. Despite a decreased coherence due to a larger separation distance, smaller positioning errors for the larger baselines $(30 \mathrm{~m})$, when compared to the small one $(0.5 \mathrm{~m})$, were found. It should be noted that this was the case even though no compensation for the Doppler shifts between the sensors connected to the large baselines was made. Also, the broadside case was used where the highest Doppler shifts occur. If an end-fire case had been used instead, even larger position errors for the smaller baselines would have been found.

The propagation characteristics in underwater acoustics are complicated and associated with multipath, reverberation, and changing sound speed throughout the water volume [9]. This will in turn also decrease the coherence. By using the Generalized Cross Correlation function (GCC) [10] weighted by the Phase Alignment Transform (PHAT) [10], all frequencies are given the same influence, and the coherence can thus be increased.

In sparse arrays, the same sound source is measured from different directions through different paths. This leads to a suppression of the multipath effects.

In sparse arrays, the hydrophone measured signals for the whole system can contain different sounds from the same target. The vessel sound different depending on which direction it travels in. With the help of a sparse array, all sources may be detected and imaged.

If the sound source is moving, the signals also get compressed or spread in time due to Doppler. For normal linear arrays, the Doppler shift is essentially identical all over the aperture. In sparse arrays, however, this is not the case, and as the integration time increases, the Doppler has to be compensated in each sensor correlation. By compensating for Doppler, the coherence is improved and more sensor pairs can then contribute to the image.

In this article, we have used target speed as determined by the help of a GPS instead of estimated from measurement data. The main focus of this paper is on the evaluation of the method and therefore no Doppler estimation is considered.

With high SNR of the signals, Doppler can directly be estimated from the data, and this is also one of the advantages of a spatially large sensor system. Another advantage of the system is that Doppler also discriminates against sound sources that travel at other speeds than that of the target.

\section{SignAL MODEL}

Let $s(t)$ be the signal emitted from a target sound source. The received signal $\tilde{x}_{n}(t)$ at hydrophone $n$ at measurement time $t$ can then be modeled as

$$
\tilde{x}_{n}(t)=s\left(\tilde{D}_{n}\left(t-\delta t_{n}\right)\right)+n_{n}(t)
$$

where $\delta t_{n}$ is the propagation time from target to hydrophone $n, \tilde{D}_{n}$ is the signal compression or widening connected to the movement of the sound source, and $n_{n}(t)$ is uncorrelated noise. The projected velocity to each sensor and its connected Doppler will, in a sparse array, be very different, resulting in separate compression ratios for each sensor.

In a scattered array, the sensors have different positions, and a target will therefore likely maneuver inside of the lattice of the array. This means that the array elements may cover up to 360 degrees of the target. In the scattered array, different sensor pairs will be situated in different directions in relation to the sound source. The opening angle, $\phi_{m n}$, for the sensor pair $m n$ with the baseline $b_{m n}$ (distance between sensors) in comparison to the sound source is defined as shown in Fig. 1.

In a passive system with a sparsed array, large values of the angle $\phi_{m n}$ will limit the coherence in the sound field between the two measurement points. This is not considered in (1) but is discussed in Section IV-E, where different bandwidths are chosen to suit the opening angle of each sensor pair.

\section{A. Doppler effects}

If a sound source is moving to or from a sensor, it causes the received signal to become either decompressed or compressed in time. This means that the frequency shifts as a sound moves 


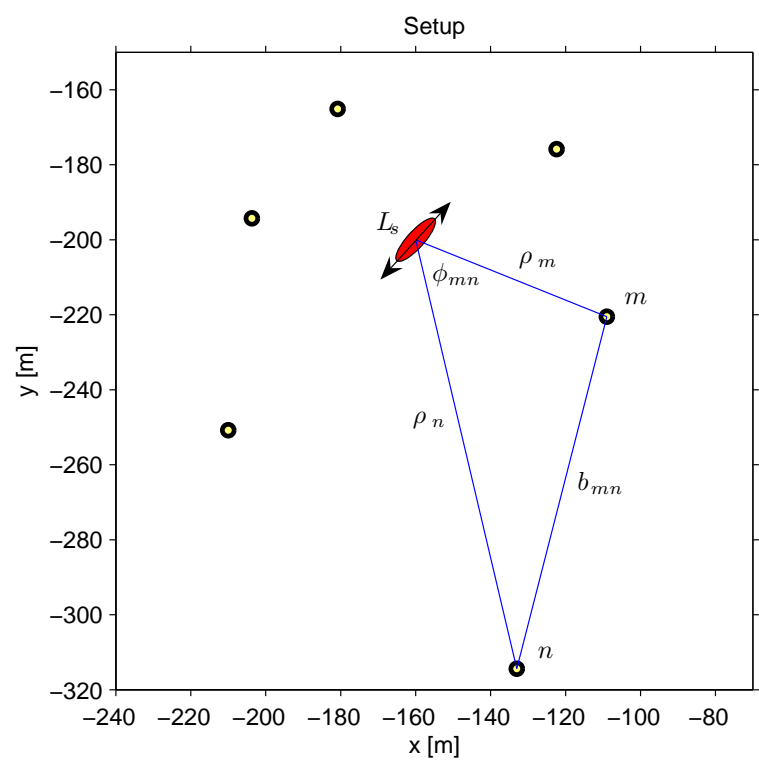

Fig. 1. Example of setup where geometric parameters are defined.

away from a sensor, and there will be a decrease in frequency. If the sound source moves closer, there will be a increase in frequency.

In a scattered sensor system, this can be used to estimate the velocity and moving direction. For setups with only large baselines and signals of low SNR, blind hypotheses have to be tested until detection can be made. However, with high SNR of the signals, Doppler can directly be estimated from the data, and this is also one of the great advantages of a spatially large sensor system. It is also necessary to compensate for the Doppler effect when correlating different sensor pairs to increase the peak to noise ratio.

In [7], Doppler is found using the ambiguity function and is valid when acceleration is negliable. To show how the ambiguity function can be used to find the Doppler, and thereby the velocity and speed, Fig. 2 plots the result of this function from our own data set. A clear maxima is seen for a Doppler correction of -0.015 percent of $\tilde{x}_{5}(t)$ compared with $\tilde{x}_{6}(t)$ at the pairwise delay $(\tau)=-3.42 \mathrm{sec}$. The ideal values given from simulation of the same setup are nearly identical $(-0.015$ percent, $-3.5 \mathrm{sec})$.

The work in this article focuses yet on an imaging process, so pre-known speed is therefore used in the process. The following pre-processing is performed to transform all the sensor signals to zero Doppler.

$$
\begin{aligned}
x_{n}(t) & =\tilde{x}_{n}\left(D_{n}(t)\right) \\
& =s\left(D_{n}\left(\tilde{D}_{n}\left(t-\delta t_{n}\right)\right)+n_{n}\left(D_{n}(t)\right)\right. \\
& =s\left(t-\delta t_{n}\right)+\tilde{n}_{n}(t)
\end{aligned}
$$

where $\tilde{n}_{n}(t)$ is considered to be uncorrelated noise and $D_{n}(t)$ is the transfer function to get back to zero Doppler. If no compensation for Doppler is done, it results in a decreased

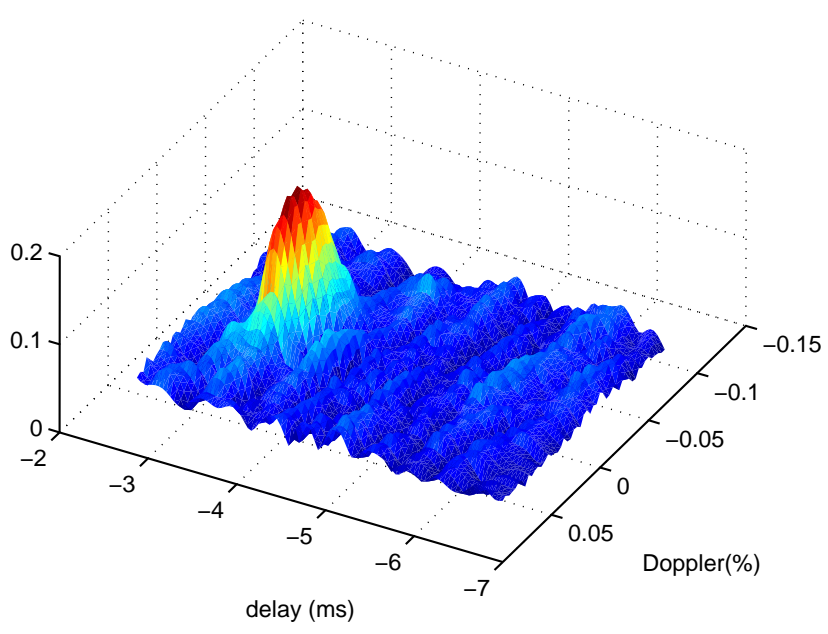

Fig. 2. Ambiguity function for sensor pair 56 in Setup 1B, where intergration time is $1 \mathrm{sec}$.

TABLE I

TARGET, ENVIRONMENT, AND SENSOR SETUPS FOR THE DIFFERENT MEASUREMENTS WHERE THE SOUND SPEED IS GIVEN AS $c$ AND DEPTH IS SENSOR DEPTH.

\begin{tabular}{c||r|r|r|r|r}
\hline Setup & Target & Bottom & $c[\mathrm{~m} / \mathrm{s}]$ & $b_{m n}[\mathrm{~m}]$ & Depth [m] \\
\hline \hline 1 & 1 & rocks & 1421 & $17-181$ & $7-12$ \\
2 & 2 & mud & 1485 & $90-141$ & $7-12$ \\
\hline
\end{tabular}

TABLE II

SIZE, SPEED, AND PLACEMENT OF TARGETS.

\begin{tabular}{c||r|r|r|r}
\hline Target & Size $[\mathrm{m}]$ & Speed $[\mathrm{m} / \mathrm{s}]$ & Propellers & Placement \\
\hline \hline 1 & $20 \times 8.0$ & 2.5 & 2 & surface \\
2 & $4 \times 1.5$ & 0 & 1 & surface \\
\hline
\end{tabular}

coherence. As $\phi_{m n}$ increases, the coherence between two sensors decreases. This was illustrated in [8], where the magnitude squared coherence, without Doppler compensation, is shown for different sizes of baselines and thereby for different Doppler. Another potential of a sparse large array, where large Doppler can be found, is that Doppler discriminates against sound sources with other speeds than that of the target.

\section{MEASUREMENT DATA}

\section{A. Measurement setup}

In this article, we present and analyze results from two different measurement campaigns with two different measurement setups and three different measurements. The measurement setups and target properties are given in Table I and II. In Fig. 3 and Fig. 4, an illustration of the three different measurement setups are shown.

For the two first measurements, $1 \mathrm{~A}$ and $1 \mathrm{~B}$, the same target is used. The target - sensor distance is, however, generally bigger than the baseline $b_{m n}$ in $1 \mathrm{~A}$, while in $1 \mathrm{~B}$ the baseline is bigger than the distance. In Measurement $1 \mathrm{~A}$, the target is far away from the coastline while in $1 \mathrm{~B}$ the coastline, is $\sim$ $150 \mathrm{~m}$ on the opposite side of the target in comparison to the sensor network. In Measurements $1 \mathrm{~A}$ and $1 \mathrm{~B}$, the target is 
moving with a speed of $2.5 \mathrm{~m} / \mathrm{s}$, while in Measurement 2 the target is not moving. According to Tables I and II, the target in Measurement 2 is very small in comparison to the target in Measurement 1. Note that the bottom condition with rocks in Setup 1 gives stronger bottom reflection than in Setup 2 where the bottom is covered with mud, as is shown in Table I. During the experiment, the positions of sensors and targets were measured with a Differential Global Positioning System (DGPS) which gives a deviation of 0.5 to $5 \mathrm{~m}$.

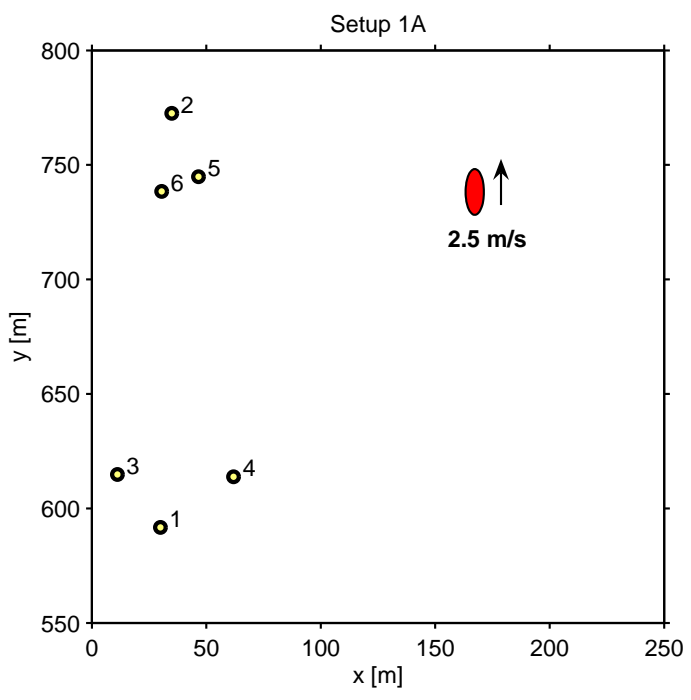

a) Measurement $1 \mathrm{~A}$

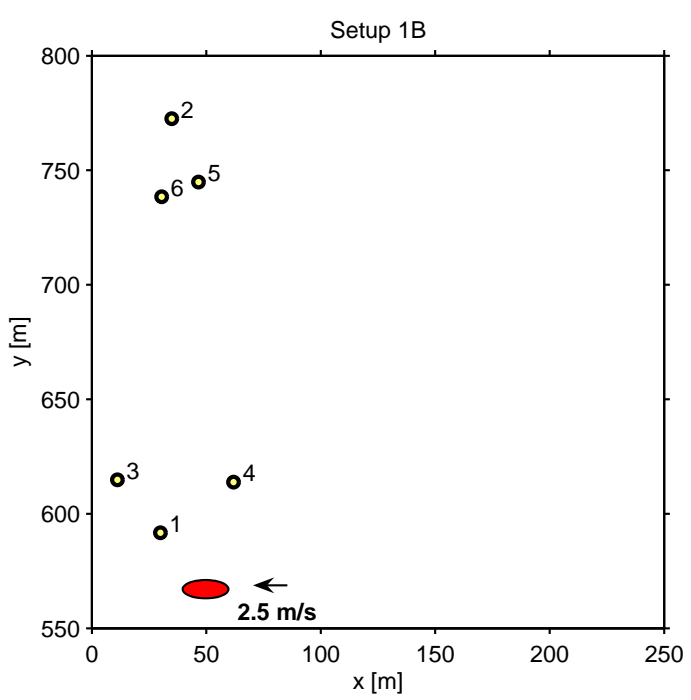

b) Measurement $1 \mathrm{~B}$

Fig. 3. Setup 1. In Measurement 1A, the distance to all sensors is approximately the same, while in Measurement 1B the distances are very different. The water environment is given in Table I and target properties are given in Table II

\section{B. Target sound}

Frequency spectra of the received signals in Setup 1 are shown in Fig. 5 and Fig. 6, and in Fig. 7 only an estimate of the frequency spectra of one sensor is shown for Setup 2

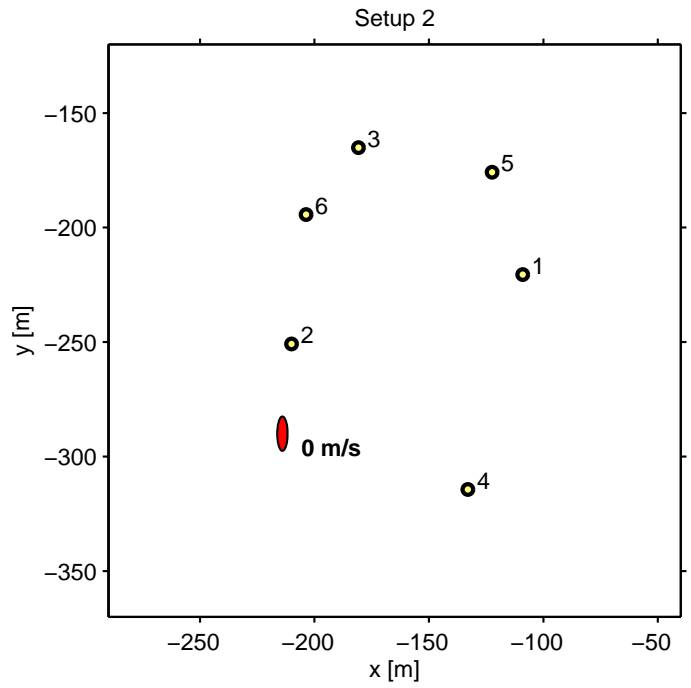

Fig. 4. Setup 2. Sensor to target distances are smaller or in the same size as the sensor baselines. The water environment is given in Table I and target properties are given in Table II.

because the sensor signals were not satisfactorily calibrated in this experiment. This absolute calibration error is, however, not important for the final result, since PHAT weighting of the GCC will normalize all frequencies resulting in an amplitude equal to one in the frequency plane. The frequency spectra in Fig. 5 and Fig. 6 are from the same target and therefore the spectra are similar. They decay in both setups as the frequency increases. However, there is a broad peak at 10 $\mathrm{kHz}$ for Measurement 1A in comparison to $1 \mathrm{~B}$. A reasonable explanation for the peak in $1 \mathrm{~A}$ could be that the stern of the target is sensed by several sensors and in this way the noise from the cavitation of the two propellers is registered.

In spectra for Measurement $1 \mathrm{~B}$, there is an indication that tonal components (higher levels in $1-9 \mathrm{kHz}$ ) are registered from the target. This can also can be confirmed by the sound files. In Measurement 1B, the engine sound could clearly be heard, while in Measurement $1 \mathrm{~A}$ the engine could not be heard. The maximum bandwidth of $12 \mathrm{kHz}$ will be used for both Setups, and the effective bandwidth is analyzed in Section IV-E.

\section{IMAGING}

The sensors and targets are placed in the 3D space. In this article, the images are for the sake of convenience processed for one depth (surface), which results in a two-dimensional (2D) image. This is also consistent with the two surface vessels used as targets in the measurements. An image is defined as the pairwise coherence projected over the area of interest.

\section{A. Cross-correlation}

In this work, we use the GCC function $R_{m n}(\tau)$ between two sensor signals $x_{m}$ and $x_{n}$, where

$$
R_{m n}(\tau)=\int_{-\infty}^{\infty} W_{m n}(f) G_{m n}(f) e^{j 2 \pi f \tau} d t
$$




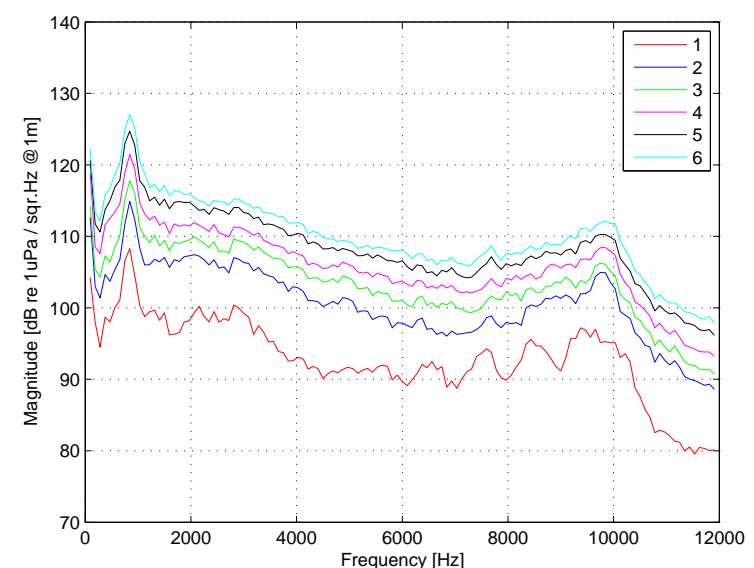

Fig. 5. Spectra estimate for all sensor signals in Measurement 1A. In the estimate, data of $1 \mathrm{sec}$ are divided into 94 averages where boxcar is used. The frequency resolution $\Delta \mathrm{f}$ is $94 \mathrm{~Hz}$.

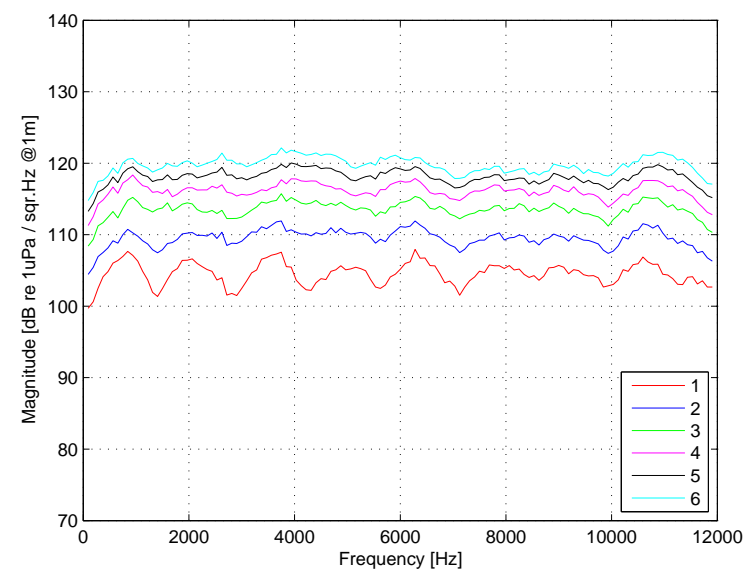

Fig. 6. Spectra estimate for all sensor signals in Measurement 1B. In the estimate, data of $1 \mathrm{sec}$ are divided into 94 averages where boxcar is used. The frequency resolution $\Delta \mathrm{f}$ is $94 \mathrm{~Hz}$.

and $G_{m n}(f)$ is the cross-spectra and $W_{m n}(f)$ is a weighting function. If the broadband signal contains dominant frequency components, as in Figs. 5 - 7, this can cause spatial aliasing. A weighting function, $W_{m n}(f)$, is used to manipulate the importance of the different frequencies. The PHAT-weighting function [10] takes away effects from dominant tones by weighting all frequencies equally. $W_{m n}(f)$ for the PHAT weighting is given by

$$
W_{m n}(f)=\frac{1}{\left|G_{m n}(f)\right|} .
$$

As described in Section IV-E, the coherence depends on the bandwidth used, and to compensate for that, a filter function, $P(f)$, is used. The total overall weight is then defined as

$$
W_{m n}(f)=\frac{P(f)}{\left|G_{m n}(f)\right|} .
$$

In some measurements, the peak of the GCC function is negative. Negative dips near a peak could, for example, be

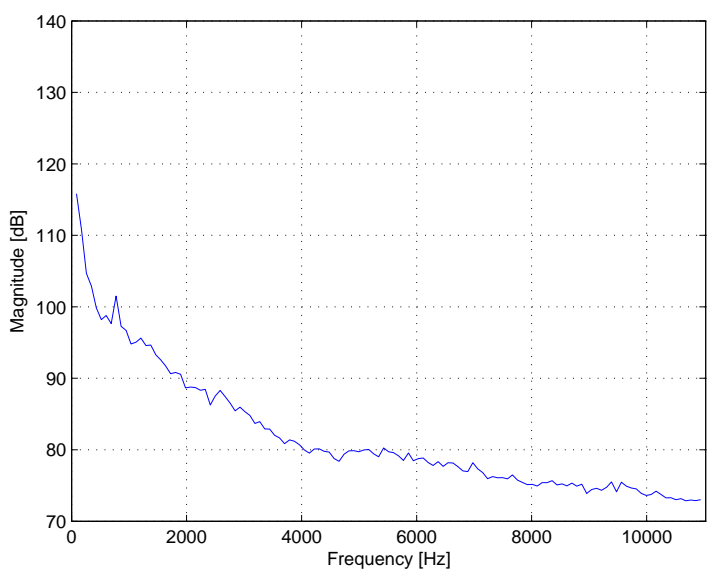

Fig. 7. Spectra estimate of sensor signal 2 in Measurement 1B. In the estimate, data of $1 \mathrm{sec}$ are divided into 86 averages where boxcar is used. The frequency resolution $\Delta \mathrm{f}$ is $86 \mathrm{~Hz}$.

caused by a sound source that is beneath the surface. By using the envelope of $R_{m n}(\tau)$, the negative peaks are turned positive, and a broader peak of $R_{m n}(\tau)$ is given. This is favorable for low resolution images and is also more robust against sensor position errors, as described in Section IV-D. We call this non-coherent processing, and it is defined as

$$
\tilde{R}_{m n}(\tau)=\left|R_{m n}(\tau)+j \mathcal{H}\left(R_{m n}(\tau)\right)\right|
$$

where $\mathcal{H}$ is the Hilbert transform of $R_{m n}(\tau)$ and is implemented as in [11].

\section{B. Image discretization}

The area where an image is formed is divided into volumes, $\mathbf{v}_{i j k}$, in which the cross-correlation sample, $R_{m n}\left(\tau_{m n i j k}\right)$, is backprojected. The time delay, $\tau_{m n i j k}$, in each correlation is given by

$$
\tau_{m n i j k}=\frac{\left|\mathbf{p}_{m}-\mathbf{v}_{i j k}\right|-\left|\mathbf{p}_{n}-\mathbf{v}_{i j k}\right|}{c}
$$

where $c$ is the speed of sound, and $\mathbf{p}_{m}$ and $\mathbf{p}_{n}$ are the sensor positions. In (7), we have approximated the sound speed $c$ as constant for all positions within the volume. This is a good approximation for our three measurements as shown in Section IV-C. In a general case, however, positions calculated from the time delay have to consider different sound speeds in different areas of the water volume. This is especially true for areas at a greater distance from the sensors.

The cubic image or map, Map, is formed as

$$
\operatorname{Map}\left(V_{i j k}\right)=\sum_{m=1}^{N-1} \sum_{n=m+1}^{N} R_{m n}\left(\tau_{m n i j k}\right)
$$

where $N$ is the number of the sensor pair.

The sampling in time of the signal gives the discretization, $d a_{m n}$, in the spatial domain. The indexation $m n$ of the different discretizations is given from each sensor pair bandwidth given in Section IV-E. 


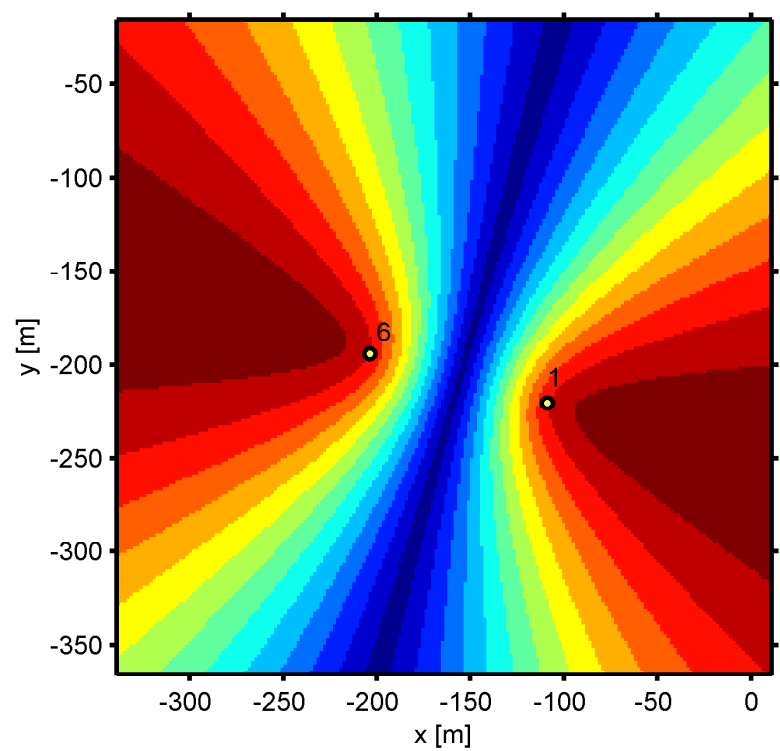

Fig. 8. The image discretization shown for sensor pair 16 in Setup 2. The $\mathrm{z}$-plane is at the surface, $\mathrm{z}=0$. The two dots are the sensors. Each integer value of $\tau$ give rise to a hyperbolic shape of several positions in space, here shown by colors. Dark blue is $\tau=0$ and dark red is $\tau=\max$.

Between two sensors, the time delay $\tau$ is associated with a hyperboloid of possible target positions in three dimensions (when $\tau$ is projected into the spatial domain). In Fig. 8 a twodimensional example of the spatial domain is shown. Based on the Nyqvist criteria, the maximum 2D discretization, $d x$, $d y$ for the global coordinate system, $(\hat{x}, \hat{y}, \hat{z})$, was formed in [12].

In Fig. 9, the 2D projection of a hyperboloid (given from a certain $\tau$ ) to a plane given by a sensor-dependent coordinate system, $\hat{\xi}_{m n}$ and $\hat{\eta}_{m n}$, at height $\mathrm{z}$ is shown. The sensordependent coordinate system has a unit vector, $\hat{\xi}_{m n}$, in the direction of the baseline, and $\hat{\eta}_{m n}$ is given to be orthogonal to $\hat{\xi}_{m n}$ and $\hat{z}$. We have then assumed that the global $\hat{z}$ direction is orthogonal to the baseline, which is approximately the case for our measurement setup. However, this is easily changed for any setup, though the equations will be a little bit more complicated. These equations will give us an approximate estimate of the discretization in the global coordinate system as shown in Fig. 9 and given by

$$
\begin{gathered}
d x(x, y, z)=\min \left|\frac{\delta_{m n}(x, y, z)}{\cos \left(\theta_{m n}+\alpha_{m n}(x, y, z)\right)}\right| \\
d y(x, y, z)=\min \left|\frac{\delta_{m n}(x, y, z)}{\sin \left(\theta_{m n}+\alpha_{m n}(x, y, z)\right)}\right|
\end{gathered}
$$

where $\delta_{m n}(x, y, z)$ is the distance between the two discretized hyperbolas at the point $(x, y, z) . \theta_{m n}$ is the rotation angle between the coordinate system $\left(\hat{\xi}_{m n}, \hat{\eta}_{m n}\right)$ and $(\hat{x}, \hat{y})$. The rotation angle $\alpha_{m n}(x, y, z)$ is the angle between $\hat{\delta}_{m n}(x, y, z)$ and $\hat{\xi}_{m n}$. $\hat{\delta}_{m n}(x, y, z)$ is the unity vector of $\delta_{m n}(x, y, z)$.

As a consequence of (9) and (10), the sampling distance decreases as the target-to-sensor distance increases in comparison

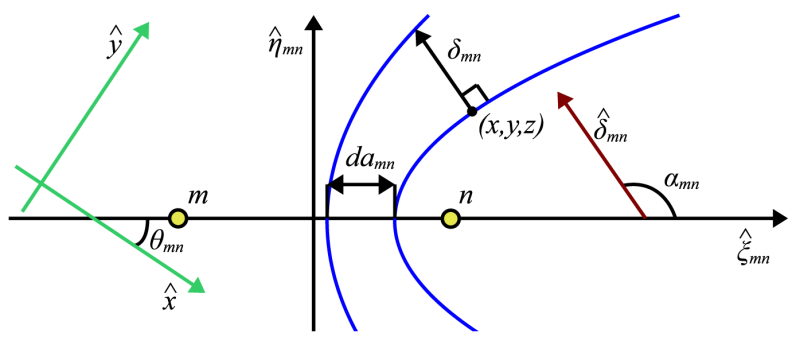

Fig. 9. The discretization, $d a_{m n}$, for sensor pair $m n$ (yellow dots) is connected to a hyperbola in the coordinate system $\left(\hat{\xi}_{m n}, \hat{\eta}_{m n}, z\right)$ with the origin of the coordinates in the middle between the two sensors. Coordinate system $(\hat{x}, \hat{y}, \hat{z})$ is the global coordinate system for all sensors with its origin at an arbitrary point which can be different from the local coordinate systems.

to the baseline. This relation can be used to save computational load but is not investigated in this article.

\section{Sound propagation}

The cross-correlation, $R_{m n}(\tau)$, is transformed into positions through the sound speed. Therefore, the true sound speed is very important. During the experiment, the true sound speed was measured for different depths. In Measurement 1, the measured sound-speed profile showed almost constant speed of sound with depth, $1421 \mathrm{~m} / \mathrm{s}$. However, in Measurement 2, a more complicated sound-speed profile was found (Fig. 10).

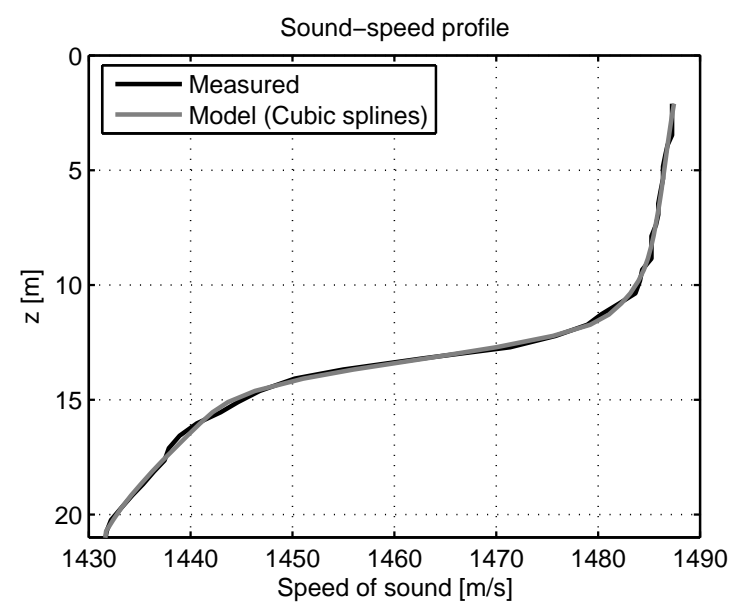

Fig. 10. Model and measured sound-speed profile for Measurement 2, where the surface is at $\mathrm{z}=0$.

In this case, the decreasing sound speed with depth causes the sound rays to bend downwards toward the bottom [9]. To calculate the effect of the sound propagation, a ray tracing model [13] was used according to

$$
\frac{d^{2} x}{d z^{2}}=-\frac{c\left(z_{0}\right) c(z)}{c^{\prime}(z) \cos \beta_{0}}
$$

where $c(z)$ is the sound speed, $z_{0}$ is the depth of the sound source and $\beta_{0}$ is the initial angle of ray from the sound source ( $\beta_{0}=0$ is perpendicular to $\mathrm{z}$ ). In the calculations, we used a model of $c(z)$ based on 11 non-equidistant cubic splines. 
TABLE III

DIFFERENCE BETWEEN TWO MODELS OF SOUND PROPAGATION FOR MEASUREMENT 2. ONE ASSUMES THE SOUND SPEED TO $1485 \mathrm{M} / \mathrm{s}$. THE OTHER IS BASED ON RAY TRACING CALCULATED FROM THE MODEL IN FIG. 10.

\begin{tabular}{|c||r|r|r|}
\hline Sensor & Range $[m]$ & Diff $[m]$ & Depth $[m]$ \\
\hline \hline 1 & 138 & 0.05 & 13 \\
2 & 57 & 0.01 & 13 \\
3 & 144 & 0.05 & 13 \\
4 & 85 & 0.02 & 13 \\
5 & 114 & 0.04 & 12 \\
6 & 162 & 0.21 & 18 \\
\hline
\end{tabular}

Propagation delays calculated from the model with bending rays were compared with straight rays calculated by a sound speed of $1485 \mathrm{~m} / \mathrm{s}$ for Measurement Setup 2. The differences in Measurement 2 were only a number of centimeters as shown in Table. III. Since the estimate of the sensor position has lower accuracy than the difference presented in Table III, the soundspeed profile was not taken into account. A fixed sound speed of $1485 \mathrm{~m} / \mathrm{s}$ is therefore used in Measurement 2 .

Multipath is not considered to be a problem within the sensor network. Each sensor pair registers a different multipath and those will average out in the image, unlike the true sound source that will add coherently from the contributions of each sensor pair.

\section{Sensor position accuracy}

The systems described in this article give high resolution and accuracy. In the near-field even 3D resolution can be found. However, near-field processing is sensitive to errors of the sensor positions.

Since each sensor pair map is projected on top of each other, it is important that each peak from $R_{m n}(\tau)$ in the map intersects with the others at the correct positions. Otherwise, the cross-correlations will not add together coherently.

This is very important since the peak in the cross-correlation function is close to the sinc function if a boxcar filter is used. Negative dips will contribute destructively in the image.

If the positions of the sensors are incorrect, this will cause a situation where the target will be unfocused and smeared, as described in Fig. 11. This will lead to a decreased detection capability and loss in image quality.

In this case, non-coherent processing as described in Section IV-A can be used to eliminate some of these effects caused by small sensor displacement. If permitted by the sound substance, another solution is to use a lower bandwidth. However, this will lead to reduced performance.

A certain error in the sensor position will cause a crosscorrelation pulse (a certain time delay in a sensor pair) and its corresponding hyperbola in space, to move within the same size as the error of the sensor position. From Fig. 8 , we can also see that hyperbolas given from maximum time delays in a sensor pair (end-fire of a sensor pair in relation to sound source) corresponds to a wider area and will be drawn by a fatter line than hyperbolas drawn for zero delay (broadside sensor pairs). From this, it can also be concluded that broadside sensor pairs are more sensitive to

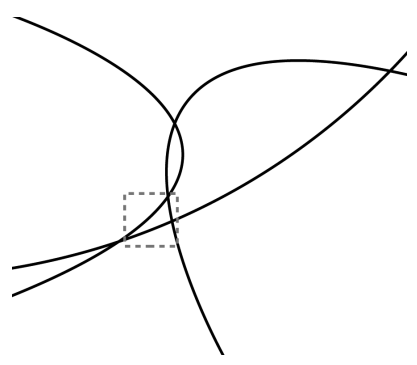

a) Unfocused

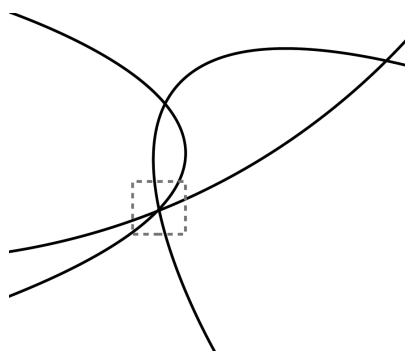

b) Focused
Fig. 11. Example of three $R_{m n}^{\max }(\tau)$ that gives rise to three hyperbolas in the $2 \mathrm{D}$ space. To get the true position and a focused map of the sound source, it is important to get the hyperbolas from all sensor pairs to summarize into one point. If there exist errors in the sensor positions, the hyperbolas will not be summarized into the correct point and the image might be unfocused.

sensor position errors. The height resolution is also better for end-fire sensor pairs which is important since the sound source point is focused at the right depth only. This gives the system better resolution in the near-field, but it then demands that the sensor positions are known more pricesely. This leads to the conclusion that errors in the sensor positions are more likely to give unfocused images in the near-field than in the far-field.

\section{E. Sensor pair bandwidth}

Since all possible combinations of sensor pairs are used, it is important to choose a bandwidth that suits the baseline of the sensor pair.

Despite the fact that the target has a certain bandwidth, there is also a geometric-dependent effective bandwidth. It is especially important to choose a proper bandwidth to improve the coherence since we use GCC-PHAT which weights all frequencies, within the sensors bandwidth, equally.

To get the geometric effective bandwidth, we assume that a sound source has an extension, $L_{s}$. At wavelength $\lambda$, the sound source will have a lobe width approximately given by $\lambda / L_{s}$. This is a known relation in wave physics, and it is valid in the far-field of the sound source and when $L_{s}$ is larger than $\lambda$.

If the two sensors $m n$ with a separation $b_{m n}$ should be in the main lobe of the sound source, the minimum wavelength has to be

$$
\lambda_{m n} \sim \frac{b_{m n} L_{s}}{\rho_{m n}}
$$

where $\rho_{m n}$ is the distance between the sensors and the target. If we assume that $\rho_{m n}$ is approximately the same for all sensor pairs, the maximum frequency for pair $m n$ is given by

$$
f_{m n}^{\max } \sim f^{\max } \frac{b_{m n}^{\min }}{b_{m n}}
$$

where $b_{m n}^{\min }$ is the smallest sensor pair baseline and $f_{m n}^{\max }$ is the maximum frequency used in analysis. In this equation, no assumptions about the position or size of the sound source have to be considered to find a suitable bandwidth. In Setup 1A, the distance from the sensor pairs to the target is approximately the same. Thus, (13) is chosen for this case. In Table IV, the bandwidth for each sensor pair is shown. 
TABLE IV

SETUP 1A, $f_{m n}^{\max }$ CALCULATED FROM (13).

\begin{tabular}{|c||r|r|}
\hline Sensor pair [mn] & $b_{m n}[m]$ & $f_{m n}^{\max }[H z]$ \\
\hline 12 & 181 & 1127 \\
13 & 30 & 6800 \\
14 & 39 & 5231 \\
15 & 154 & 1325 \\
16 & 147 & 1388 \\
23 & 159 & 1283 \\
24 & 161 & 1267 \\
25 & 30 & 6800 \\
26 & 34 & 6000 \\
34 & 51 & 4000 \\
35 & 135 & 1511 \\
36 & 125 & 1632 \\
45 & 132 & 1545 \\
46 & 129 & 1581 \\
56 & 17 & 12000 \\
\hline
\end{tabular}

In Fig. 12, the quality of $R_{m n}(\tau)$ using Table IV is plotted to be compared to different fixed bandwidths (the same for all sensor pairs). The quality of $R_{m n}(\tau), Q_{R_{m n}}$ is measured as, $Q_{R_{m n}}=R_{m n}^{\max }(\tau) / \sigma$ where $R_{m n}^{\max }(\tau)$ is the maximum value of $R_{m n}(\tau)$ and $\sigma$ is the standard deviation of $R_{m n}(\tau)$.

In Measurement 1B and 2, the opening angles are extreme, and the approximation $\lambda / L_{s}$ is not valid. For these measurements, a fixed (the same for all sensor pair) bandwidth is used instead. In Fig. 13 and Fig. 14, results from different fixed bandwidths are shown. From the figures, it is concluded that the bandwidth $\mathrm{BW}=4 \mathrm{kHz}$ in Setup $1 \mathrm{~B}$ gives higher quality for the sensor pairs generally, and for Setup 2, BW=12 kHz is generally better.

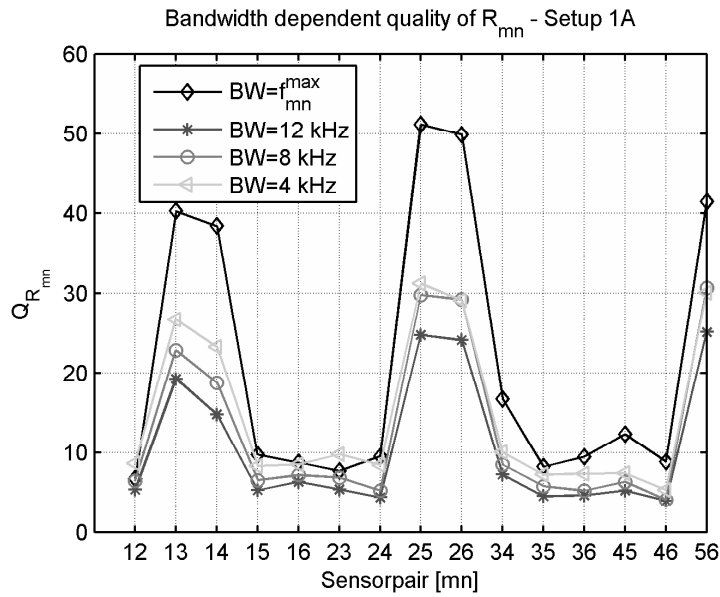

Fig. 12. Setup 1A, Peak to standard deviation in correlation result for different fixed bandwidths and a baseline-dependent bandwidth.

\section{MODEL AND METHOD IN APPLICATION}

This chapter demonstrates the application of the proposed method by using data from the three measurements. The chapter is divided into four parts, where the first part describes how the image processing is conducted, and the three following parts show the results for each measurement.

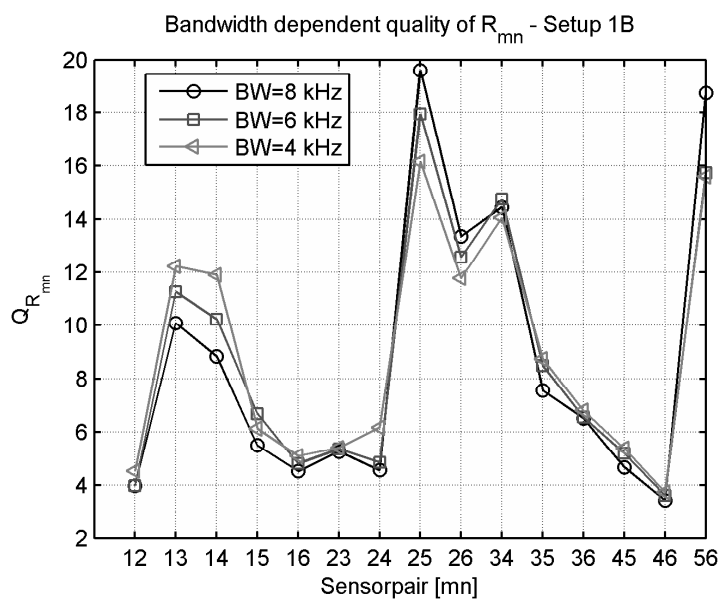

Fig. 13. Setup 1B, Peak to standard deviation in correlation result for different fixed bandwidths.

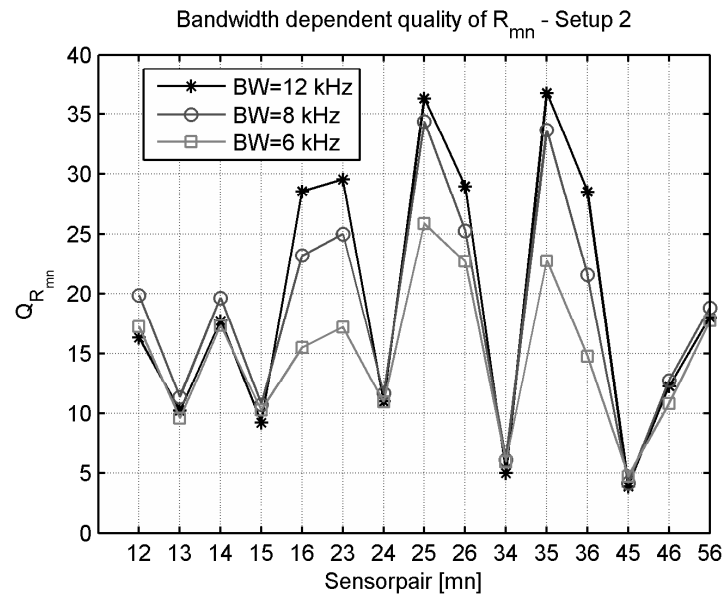

Fig. 14. Setup 2, Peak to standard deviation in correlation result for different fixed bandwidths.

\section{A. Image processing}

In this article, we present the results in six steps of different processing to show the effects from the proposed algorithms. The processing steps illustrate the performance of the algorithms for the measurement setups in the shallow waters. Measurement data for one moment in time are used and compared to simulations.

1) Step 1 - Overview: A large unfocused image of the experimental area is shown for low frequencies up to $4 \mathrm{kHz}$ with no Doppler adjustment. This image is used to show that there is not any spatial aliasing in the area. Non-coherent filtering that is associated with a wider correlation peak is used as described in Section IV-A.

2) Step 2 - Zoom: In this step, a zoomed image of the target is shown. A larger bandwidth is used in comparision to step 1, since the volume elements are smaller. Non-coherent processing is used, and no Doppler adjustment is performed.

3) Step 3 - Doppler: This image is Doppler adjusted with the pre-given Doppler, calculated from the GPS onboard the ship. More sensor pairs can then contribute to the image, and the image becomes more focused. 
4) Step 4 - Coherent processing: In this step, both coherent processing and Doppler adjustment is used in the processing. Coherent processing gives a higher resolution in the image of the sound sources, if the sensors are placed at the pre-assumed positions. It also increases the signal to noise ratio.

5) Step 5 - Baseline-dependent bandwidth: For this image, the sensor pair-dependent bandwidths as described in Section IV-E are used to get better correlation results. This step is done for Setup 1A only.

6) Step 6 - Simulation comparison: To compare the result with an ideal situation, simulations are performed. Dependent on the assumed properties of the target, one or two broadband sound sources are simulated. The sources are simulated as point-like white noise sources. In the simulation, the coherent method with Doppler adjustment is used.

\section{B. Results from Setup $1 A$}

For Setup 1A, the target is passing at a distance $<100$ $\mathrm{m}$ from the sensors. The course of the target is such that it is facing some sensor pairs with its stern. As seen in Fig. 12, the cross-correlation is clearly separated from the surrounding noise. The target can be detected in all different steps suggested in Section V-A. Even the two propellers at the target, spaced $\sim 4 \mathrm{~m}$ apart, can be separated in Step 5, which is seen in Fig. 16f. This Figure also agrees with the simulated image in Fig. 16g.

1) Step 1 - Overview: A rough estimate of the target position can clearly be seen in the overview map in Fig. 15a.

2) Step 2 - Zoom: Fig. 15d is zoomed in, according to the darker area in Fig. 15a. The image is blurry due to the incorrect Doppler.

3) Step 3 - Doppler: In this step, Doppler compensation is done, and by comparing Fig. 15d and Fig.15g, it is confirmed that adjustment of Doppler in the sensor signals gives a more focused image.

4) Step 4 - Coherent processing: Coherent processing gives higher resolution. The sound source in setup $1 \mathrm{~A}$ can be seen with higher accuracy in Fig. 16b then if only Doppler adjustment is used as in Fig. 16c.

5) Step 5 - Baseline-dependent bandwidth: By applying the baseline dependent bandwidth, the target's two sound sources can be separated, as seen in Fig. 16f. This clearly indicates that sparse arrays can separate targets in the range direction using mainly low frequencies. This is consistent with the higher correlation peaks as shown in Section IV-E.

6) Step 6 - Comparison with simulation: In Fig. 16g, a simulation of Setup 1A with two moving sound sources is shown. Processing is conducted as in Fig. 16f. The simulation image is similar to the image in Fig. 16f. The possibility of imaging the two sound sources is thereby verified by simulation.

\section{Results from Setup $1 B$}

The target is the same as in Setup 1A. In this case, however, the target is in another position very close and is observed from the side. The two propellers, spaced $\sim 4 \mathrm{~m}$ apart, can be assumed to be seen as one larger sound source. The envelope,
$\tilde{R}_{m n}(t)$, in Fig. 16 b gives the best result. This probably arises from non-accurate sensor positions, which also is indicated in the overview image (the most distinct hyperbolas are crossed in several points). Another interpretation is that this setup is more complicated than the others, with a decreasing depth behind the vessel resulting in a coastline of stones $200 \mathrm{~m}$ from the vessel.

By non-coherent processing, two sound sources at the target can be seen in the image. In this setup, 1B, in comparison to the others $1 \mathrm{~A}$ and $1 \mathrm{C}$, the sound from the engine can be detected by the human ear in the recorded sound files from the most closely placed sensors. We therefore think it is very likely that the target's engine and the propeller sound are resolved in the image. This is also indicated in the spectra (Fig. 7) of this measurement, since tones of low frequencies are higher in this measurement than in the spectra of Measurement $1 \mathrm{~A}$.

1) Step 1 - Overview: In Fig. 15b, we can clearly see that a rough position estimate of the target is harder to get for this setup in comparison to setup 1A. This may be due to uncompensated Doppler, inaccurate sensor positions, or by the effect of several sound sources causing several intersection points.

2) Step 2 - Zoom: In Fig. 15e, the map is zoomed in according to the GPS. In this case, little coherent sound is seen, but still no Doppler or bandwidth adjustment is done. A diffuse image of the target is seen in Fig. 15e.

3) Step 3 - Doppler: Adjustment of Doppler in the sensor signals gives a much more focused image of the target in Fig. 15h. Two crossing points are shown which may be caused by inaccurate positioning of the sensors. However, a more reasonable explanation is that the two strong points or energy levels in the map could be an indication that the engine as well as the propellers can be imaged and resolved. As mentioned in Section III-B, the engine is clearly heard in the closest sensor signals for this setup.

4) Step 4 - Coherent processing: By using coherent processing, a more accurate image of the sound source can be found, if the sensor positions are known exactly. This is not the case for this setup, and the processing is very sensitive to small errors in the sensor positions. Lower frequencies create a broader peak in the cross-correlation function, and destructive effects from the errors of the sensor positions as described in Section IV-B in the map are seen in this image, Fig. 16d. The non-coherent processing is therefore crucial for this measurement setup.

5) Step 5 - Baseline-dependent bandwidth: Since the opening angle is very large in this case, no baseline-dependent filtering is done for this setup.

6) Step 6 - Comparison with simulation: In Fig. 16h, simulation of two point-like sound sources is shown. Noncoherent processing is conducted as in Fig 16b. The choice of non-coherent processing is due to the position error of the sensors. The simulation image Fig. $16 \mathrm{~h}$ is very similar to the measurement in Fig. 16b. This indicates that two sound sources are resolved in the target. 


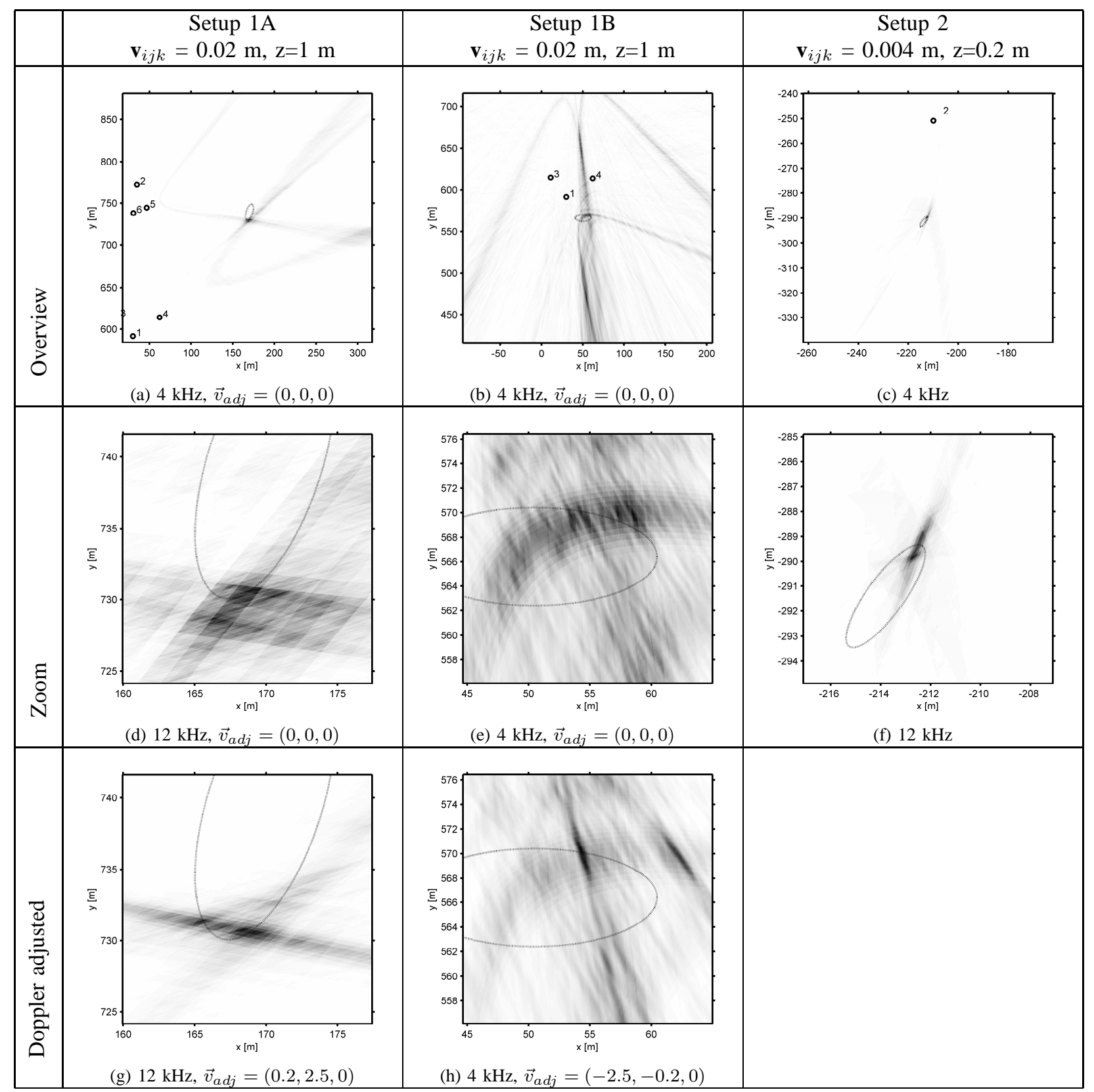

Fig. 15. Processing step 1-3, 2D-map results

\section{Results from Setup 2}

1) Step 1 - Overview: For this measurement, a focused target is already seen in Fig. 15c, since the target is nonmoving and small.

2) Step 2 - Zoom: In Fig. 15d, the map is zoomed in and the bandwidth used is $12 \mathrm{kHz}$. One sound source is clearly seen. It is, however, a bit smeared most likely due to the noncoherent processing.

3) Step 3 - Doppler: This step is not evaluated since the target is not moving.

4) Step 4 - Coherent processing: With coherent processing, a much more focused point is seen in Fig. 16e. The sound is now displayed on a slightly different place. The difference may arise from the differences in the non-coherent and coherent signal processing.
5) Step 6 - Comparison with simulation: In Fig. 16i, simulation of a point sound source is shown. A more focused point is seen in the simulation shown in Fig. 16i than in measurement Fig. 16e, which probably arises from inaccurate sensor positions.

\section{E. Conclusions}

This article shows that it is possible to position and image near-field sound sources by a scattered array. If proper Doppler adjustment is used, coherence is improved and several sound sources can be resolved. In the three experimental setups, correlation is shown for nearly all sensor pairs.

Sensor pair baselines from $17 \mathrm{~m}$ up to $160 \mathrm{~m}$ are used where the distance to the target is $20-300$ meters. The maximum 


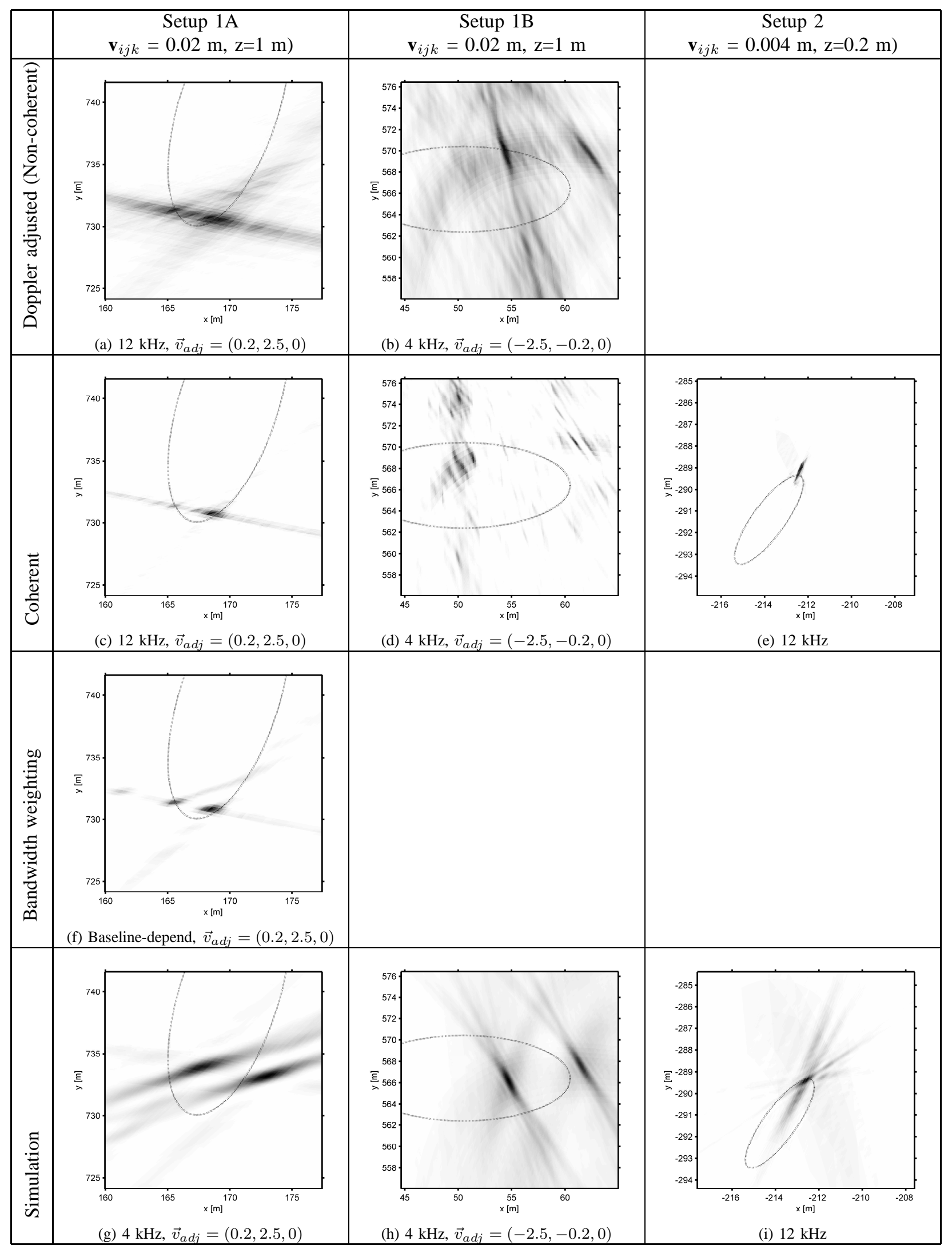

Fig. 16. Processing step $3-6,2 \mathrm{D}$-map results

water depth is $25 \mathrm{~m}$. Two targets, one $4 \times 1.5 \mathrm{~m}$ and the other $20 \times 8 \mathrm{~m}$, are imaged by the proposed method.

Yet it is shown that good accuracy of the sensor positions is necessary for near-field targets. In one measurement, the target was closer than the size of the sensor baselines. Positions near the sensors are much more sensitive to sensor position 
errors. By using a non-coherent approach, these effects can be lessened, and an image of the sound source can still be found.

The results from the measurement setups showed that the target could be imaged by the proposed method in Setup 1A, where a larger target is located at a distance comparable to the size of the sensor baselines. The two propellers at the target could be resolved in the image with high focus and resolution. In Setup 1A coherent processing was found to give the best result.

The non-coherent processing was necessary for the nearfield Setup 1B. The cause is that the larger target passed very close to some sensors, mainly end-fire pairs and many of these sensor baselines were much bigger than sensor-to-target distance. For this setup it was found that one of the propellers and the engine of the target could be resolved.

For setup 2, where a small non-moving target was located within a scattered array with only large baselines, the proposed method worked as well. The $2 \mathrm{D}$ resolution of the sound source for the setup was found to be approximately $0.5 \mathrm{~m}$.

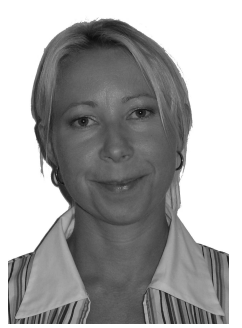

Viktoria Tegborg received the B.Sc. degree in Electrical Engineering from the Royal Institute of Technology in Stockholm in 2002 and the M.Sc. degree in Signal Processing from Blekinge Institute of Technology in 2004. Since 2005, she is a Ph.D. student at Blekinge Institute of Technology. Since 2005 Viktoria has also worked as a researcher at Swedish companies within underwater sensor electronics. At one of the companies, EmbeddedArt, she also worked with a project concerning generating underwater acoustical signatures. Presently she is Product Manager for Sonar Systems at the Swedish Defense Material Administration, who provide the Swedish Armed Forces with material, systems and methods.

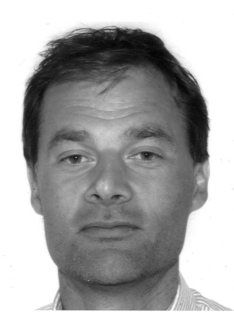

Mats I. Pettersson received a M.Sc. in Eng. Physics in 1993, a Licentiate degree in Radio and Space Science in 1995 and a Ph.D. in Signal Processing in 2000, all at Chalmers University of Technology, Gothenburg, Sweden. From 1993-1995, Mats was employed as a Ph.D. student at Department of Radio and Space Science working with radar scatterometer measurements, modeling electromagnetic scattering, and Synthetic Aperture Radar (SAR) image processing. For nearly two years, 1996-1997, he worked at Ericsson Mobile Communication in Lund as a patent project manager. From 1998 to 2007, he was employed at the Division of Sensor Technology at the Swedish Defence Research Agency (FOI) in Linköping. At FOI, he worked as a research manager and a researcher, mainly focusing on Ultra Wide Band (UWB) low frequency SAR systems and sparse radar arrays. From 2005, he has been employed at the Department of Signal Processing at Blekinge Institute of Technology. This work is related to Radar and Sonar signal processing. His main interests are SAR processing, Space Time Adaptive Processing (STAP) and underwater sparse passive arrays.

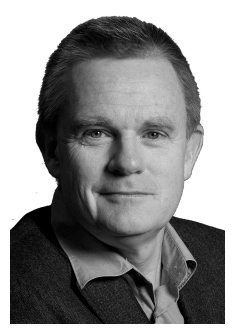

Ingvar Claesson (M'91) received the M.Sc.degree in 1980 and the $\mathrm{Ph}$. D. degree in 1986 in Electrical Engineering at University of Lund, Sweden. He was appointed Senior Lecturer in Telecommunication Theory at Lund University in 1986, and was appointed Associate Professor in 1992. In 1990, he was one of the founders of the Department of Signal Processing at Blekinge Institute of Technology, and is currently Head of Research and Principal Supervisor in Signal Processing. Since May 1998, he holds the Chair of Applied Signal Processing at Blekinge Institute of Technology, Ronneby, Sweden. He is presently also Dean at the university. His current research interests are in adaptive signal processing, blind equalization, adaptive beamforming, speech enhancement, blind signal separation, active noise control, filter design, and antenna arrays. A keen interest for applications has lead to more than 20 patents and he regularly serves as industry consultant.

\section{REFERENCES}

[1] M. Lesturgie, "Use of dynamic radar signature for multistatic passive localisation of helicopter," IEE Proceedings: Radar, Sonar and Navigation, vol. 152 , no. 6 , pp. 395 - 403, 2005. [Online]. Available: http://dx.doi.org/10.1049/ip-rsn:20050006

[2] M. Pettersson, N. Gustafsson, and P. Andersson, "Aspekter pá radarnätverk för mảlföljning av smygmảl som en kryssningsmissil pả lảghöjd." FOI - Swedish Defence Research Establishment, 2004, Linköping, Sweden, vol. FOI-R-1467SE, 2004.

[3] G. J. Elbring, H. D. Garbin, and M. D. Ladd, "Comparison of methods for 3d target localization from seismic and acoustic signatures," vol. 4040, Orlando, FL, USA, 2000, pp. 154 - 164. [Online]. Available: http://dx.doi.org/10.1117/12.392578

[4] O. Adam, J.-F. Motsch, F. Desharnais, N. DiMarzio, D. Gillespie, and R. C. Gisiner, "Overview of the 2005 workshop on detection and localization of marine mammals using passive acoustics," Applied Acoustics, vol. 67, no. 11-12, pp. 1061 - 1070, 2006. [Online]. Available: http://dx.doi.org/10.1016/j.apacoust.2006.05.013

[5] S. J. Norton, B. J. Carr, and A. J. Witten, "Passive imaging of underground acoustic sources," Journal of the Acoustical Society of America, vol. 119, no. 5, pp. 2840 - 2847, 2006. [Online]. Available: http://dx.doi.org/10.1121/1.2188667

[6] E.-M. Nosal and L. N. Frazer, "Modified pairwise spectrogram processing for localization of unknown broadband sources," IEEE Journal of Oceanic Engineering, vol. 32, no. 3, pp. 721 - 728, 2007. [Online]. Available: http://dx.doi.org/10.1109/JOE.2007.896837

[7] B. Xerri, J.-F. Cavassilas, and B. Borloz, "Passive tracking in underwater acoustic," Elsevier Signal Processing, vol. 82, no. 8, pp. 1067 85, 2002/08/. [Online]. Available: http://dx.doi.org/10.1016/S01651684(02)00240-2

[8] V. Zetterberg, M. Pettersson, and I. Claesson, "Comparison between whitened generalized cross correlation and adaptive filter for time delay estimation with scattered arrays for passive positioning of moving targets in baltic sea shallow waters," Oceans MTS/IEEE Conference "One Ocean”, Washington, DC, USA, 2005, pp. 2356 - 61.

[9] R. J. Urick, Principles of Underwater Sound, 3rd ed. McGraw-Hill, 1983.

[10] G. C. Carter, "Coherence and time delay estimation." Proceedings of the IEEE, vol. 75 , no. 2 , pp. 236 - 255, 1987.

[11] S. L. J. Marple, "Computing the discrete-time 'analytic' signal via fft," IEEE Transactions on Signal Processing, vol. 47, no. 9, pp. 2600 2603, 1999. [Online]. Available: http://dx.doi.org/10.1109/78.782222

[12] V. Zetterberg, M. Pettersson, L. Tegborg, and I. Claesson, "Passive scattered array positioning method for underwater acoustic source," Oceans MTS/IEEE Conference, Boston, MA, USA, 2006.

[13] D. Kahaner, C. Moler, and S. Nash, Numerical Methods and Software. Prentice-Hall, 1989. 